\section{A) Check for updates}

Cite this: Food Funct., 2021, 12, 2137

\title{
Entrapment of natural compounds in spray-dried and heat-dried iota-carrageenan matrices as functional ingredients in surimi gels
}

\author{
Daniel Marín-Peñalver, Ailén Alemán, M. Pilar Montero and \\ M. Carmen Gómez-Guillén (D) *
}

\begin{abstract}
Two drying methods (spray drying and heat drying) were used to entrap various natural compounds within a matrix of iota-carrageenan. The natural compounds were, namely, collagen hydrolysate $(\mathrm{CH})$, pomegranate polyphenolic extract (PP) and shrimp lipid extract (SL). The resulting dry powders were compared in terms of water solubility, entrapment efficiency, hydrodynamic particle properties, $\zeta$ potential and antioxidant properties (ABTS radical scavenging capacity, ferric ion reducing power and Folinreactive substances). Dry powders and plain compounds were incorporated into squid surimi gels, and after in vitro simulated gastrointestinal digestion (sGID), the residual antioxidant and angiotensin-converting enzyme (ACE)-inhibitory activities were evaluated. All powders showed antioxidant properties, electronegative $\zeta$ potential and great entrapment efficiency after rehydration (ranging from $\sim 70$ to $97 \%$ ). The heat-dried powders were composed of microparticles ranging from 177 to $380 \mu \mathrm{m}$ resulting in low water solubility (21.6-36.1\%), while the average particle size and solubility values of spray-dried preparations were $2.9-13.2 \mu \mathrm{m}$ and $>86 \%$, respectively. In contrast to the plain compounds, the addition of any of the microparticle dried preparations allowed obtaining well-conformed surimi gels. The ACE-inhibitory capacity of the surimi gels after SGID was increased by the addition of any of the compounds studied, but to a lesser extent by their entrapment forms (except with the entrapped SL). The antioxidant activities of gels with the entrapped compounds were even lower than those of gels without bioactives in some cases. In conclusion, the addition of dried microparticles did not increase the biological activity as compared to the plain compounds; however, they were beneficial to ensure adequate gel consistency.
\end{abstract}

Received 6th November 2020, Accepted 20th January 2021

DOI: $10.1039 /$ dOfo02922j

rsc.li/food-function phology of microcapsules, high encapsulation efficiency and extended shelf life of the resulting powders, suitability for both hydrophilic and hydrophobic compounds, processing of heat-sensitive substances with low degradation risk, low cost, and fast and energy-efficient processing. ${ }^{6}$

A variety of lipids, proteins, carbohydrates and complex biopolymer systems can be used as wall materials. ${ }^{3}$ Carrageenan, an anionic water-soluble galactose-based polysaccharide derived from the Rhodophyceae red algae, has been proposed as a microencapsulating matrix of living cells due to its capacity to form cross-linked three-dimensional networks induced by thermal/ionotropic gelation (hydrogels). ${ }^{7}$ Depending on the number and position of ester sulphate groups, carrageenan can be classified into three major types: i.e. iota, kappa and lambda. Iota-carrageenan in aqueous solution forms soft elastic gels, which are more resistant to syneresis than gels formed with kappa-carrageenan. ${ }^{8}$ Both kappaand lambda-carrageenan have been widely used as pharmaceutical encapsulating matrices based on their gelling properties or their capacity to produce complexes with active molecules. $^{9,10}$ Iota-carrageenan conjugated with soy-protein 
isolate through the Maillard reaction has been proposed as a bacterial encapsulating system obtained by a combination of spray drying and dry heating. ${ }^{11}$ However, unlike kappa-carrageenan, reports on iota-carrageenan as a microencapsulating agent of natural compounds for food applications are not available.

Carrageenans are commonly used as food ingredients due to their stabilizing, gelling, texturizing and water binding capacities. ${ }^{12}$ In particular, iota-carrageenan has been shown to form a reticular structure when included in heat-induced fish and squid mince gels, favouring protein gelation and water binding properties; ${ }^{13,14}$ however, there is no information regarding its incorporation into surimi gels in the form of microcapsules or complexes containing natural bioactive compounds. In this sense, surimi gels could be excellent matrices for the development of fish-derived functional foods, since they form highly digestible protein gel networks, which can incorporate a variety of natural bioactive compounds. ${ }^{15}$ In the previous work carried out by Marín et al. ${ }^{15}$ various food waste compounds (collagen hydrolysate from squid tunics, polyphenolic extract from pomegranate peel and albedo, and shrimp lipid extract) were encapsulated in soy phosphatidylcholine liposomes and incorporated into squid surimi gels, providing a notable radical scavenging capacity after in vitro simulated gastrointestinal digestion. These natural compounds, which were chosen for their different chemical nature and biological properties, can show different susceptibilities to thermal degradation during gel processing, or to changes in $\mathrm{pH}$ and action of digestive enzymes after oral intake, as well as different entrapment abilities depending on the encapsulation method used.

As a follow-up to the work mentioned above, in the present study different microcapsules containing the above-mentioned natural compounds were prepared by spray-drying (SD) using iota-carrageenan as the wall material. Alternatively, based on the ability of iota-carrageenan to form soft gels at elevated temperature, complex dry powders were also obtained in a more simple way, by drying aqueous mixtures of iota-carrageenan and natural compounds by forced convection at $80{ }^{\circ} \mathrm{C}$ (HD). This method for entrapment of bioactives could be an interesting alternative due to its simplicity and ease of implementation in SME industries dedicated to on-demand food production. Both types of entrapment systems were evaluated in terms of water solubility, entrapment efficiency, hydrodynamic particle properties and antioxidant capacity. Then, both dry powders and plain compounds were incorporated into squid surimi gels and subjected to in vitro simulated gastrointestinal digestion, after which the remaining antioxidant and antihypertensive activities were evaluated.

\section{Materials and methods}

\subsection{Materials}

Iota-carrageenan was obtained from Hispanagar S.A. (Burgos, Spain). Pepsin, pancreatin and ACE (EC. 3.4.15.1.) enzymes were purchased from Sigma-Aldrich, Inc. (St Louis, MO, USA). Giant squid (D. gigas) surimi was purchased from PSK Oceanos (Spain). The collagen hydrolysate $(\mathrm{CH})$ from giant squid tunics, the polyphenolic extract from pomegranate peel and albedo (PP), and the astaxanthin-containing lipid extract from shrimp cephalothorax and cuticles (SL) were produced, dried and stored at $-20{ }^{\circ} \mathrm{C}$, as reported in a previous work, ${ }^{15}$ in which their chemical compositions were also described. All reagents were of analytical grade. Milli-Q ${ }^{\circledR}$ Integral Water Purification System was used to produce pure and ultrapure water.

\subsection{Preparation of dry powders}

Iota-carrageenan was previously dissolved $(1 \% \mathrm{w} / \mathrm{v})$ in warm pure water. Then, each natural compound, $\mathrm{CH}$ (fine powder), PP (coarse powder), or SL (oil), was added in the ratio of $1: 4$ (compound/iota-carrageenan, w/w) and homogenized by vigorous magnetic stirring at room temperature for $30 \mathrm{~min}$. This core/coating ratio was selected to ensure high entrapment efficiency and stability of the resulting dried entrapment powders, as well as a balanced proportion of both iota-carrageenan and natural compound in the surimi gel. Thus, for each natural compound, two types of dry powders were obtained following two different methodologies: (i) spraydrying (SD) using a B-290 Basic Mini Spray-Dryer (Büchi, Flawil, Switzerland) with an inlet temperature of $220{ }^{\circ} \mathrm{C}$, an aspirator at $90 \%$, a pump at $65 \%$ and a $\mathrm{Q}$-flow rate of $12.3 \mathrm{~L}$ $\min ^{-1}$, denominated SD-CH, SD-PP and SD-SL; and (ii) heatdrying (HD) using a heating/drying chamber with forced convection (FD 240, Binder, Tuttlingen, Germany) at $80{ }^{\circ} \mathrm{C}$ for $20 \mathrm{~h}$ (the resulting product was ground to a fine powder), denominated HD-CH, HD-PP and HD-SL. All powders were stored at $4^{\circ} \mathrm{C}$ until use.

\subsection{Characterization of dry powders}

2.3.1. Particle size and $\zeta$ potential. The particle size was measured after dispersing the powders in distilled water by dynamic light scattering using a BI-200SM Goniometer (Brookhaven Instruments, NY, USA). $\zeta$ potential values of the dispersions were measured with a Nano-ZS ZEN 3600 Zetasizer (Malvern Instruments, Germany).

2.3.2. Entrapment efficiency. The entrapment efficiency (EE) (\%) was calculated using the following equation:

$\mathrm{EE}=($ entrapped compound/total compound $) \times 100$.

The entrapped compound was calculated from the difference between the total compound added and the nonentrapped compound, which was separated and quantified by different methods depending on the type of compound, following the procedure described by Marín et al. ${ }^{15}$

2.3.3. Water solubility. Dry powders were dispersed in distilled water $(1 \% \mathrm{w} / \mathrm{v})$ using a vortex and an AG-200 shaker (COMECTA S.A., Barcelona, Spain) at $100 \mathrm{rpm}$ for $150 \mathrm{~min}$. The dispersions were then centrifuged at $3500 \mathrm{rpm}$ and $4{ }^{\circ} \mathrm{C}$ for $5 \mathrm{~min}$, and the supernatants were collected and dried at $105{ }^{\circ} \mathrm{C}$ for $24 \mathrm{~h}$. The solubility was calculated from weight 
differences with respect to the initial sample weight and was expressed as a percentage.

\subsection{Preparation of surimi gels and in vitro gastrointestinal digestion}

Giant squid surimi was thawed and homogenized with sodium chloride $(1 \%, \mathrm{w} / \mathrm{w})$ under refrigeration and vacuum conditions using a Stephan Universal Machine (UMC 5, Stephan Machinery GmbH, Germany). The moisture level in the final product was adjusted to $80 \%$ by adding the required amount of water (in the form of crushed ice). Dry powders were added to the salt-ground surimi paste $(10 \% \mathrm{w} / \mathrm{w}$, accounting for $2 \%$ of the bioactive compound with respect to surimi) and homogenized again for $2 \mathrm{~min}$. Gels were obtained by stuffing the resulting pastes into the plastic casings with $3.5 \mathrm{~cm}$ diameter and heating at $90{ }^{\circ} \mathrm{C}$ for $45 \mathrm{~min}$. They were stored at $4{ }^{\circ} \mathrm{C}$ until use. Gels with plain compounds ( $2 \%$ with respect to surimi) and a control gel (CG) without compounds or carrageenan were prepared under the same conditions. The in vitro simulated gastrointestinal digestion (sGID) of surimi gels was carried out following the procedure described by Marín et al. ${ }^{15}$ The water soluble fraction of non-digested gels (WS) was obtained by homogenizing $20 \mathrm{~g}$ of gel with distilled water $(1: 10 \mathrm{w} / \mathrm{v}) \mathrm{using}$ an Ultraturrax mixer (IKA-Werke, Germany) and centrifuging at $10000 \mathrm{~g}$ and $4^{\circ} \mathrm{C}$ for $45 \mathrm{~min}$. The supernatant was freeze-dried and stored at $-20^{\circ} \mathrm{C}$ until use.

\subsection{Colour of surimi gels}

The Chroma (saturation) and Hue (tonality) parameters of gels were obtained as described by Marín et al. ${ }^{15}$ using a Konica Minolta CM-3500d colorimeter (Konica Minolta, Spain) equipped with a D65 illuminant and a D10 standard observer.

\subsection{Antioxidant activity}

The antioxidant activity was determined in plain compounds, dry microparticle powders and surimi gels (water soluble fraction and after sGID). The ABTS radical scavenging capacity (ABTS) and ferric ion reducing antioxidant power (FRAP) were determined as described by Alemán et al. ${ }^{16}$ Folin-reactive substances (FRS) were determined following the procedure described by Slinkard and Singleton. ${ }^{17}$ The results of the ABTS assay were expressed as $\mathrm{mg}$ vitamin $\mathrm{C}$ equivalents (VCEAC) per $\mathrm{g}$ of sample, based on a standard curve of vitamin C. The results of FRAP were expressed as $\mu \mathrm{M} \mathrm{Fe}^{2+}$ equivalents per $\mathrm{g}$ of sample, based on a standard curve of $\mathrm{FeSO}_{4} \cdot 7 \mathrm{H}_{2} \mathrm{O}$. The results of FRS were expressed as $\mathrm{mg}$ of gallic acid (GA) equivalents per $\mathrm{g}$ of sample, based on a standard curve of gallic acid. For appropriate comparison between the plain compounds and dry powders, the results were expressed per gram of bioactive compound and per $\mathrm{g}$ of dry powder.

\subsection{Antihypertensive activity}

The antihypertensive capacity was measured according to the angiotensin-converting enzyme (ACE) inhibition method described by Alemán et al. ${ }^{16}$ using a High Performance Liquid Chromatography analyser (HPLC, model SPE-MA10AVP, Shimadzu, Kyota, Japan). All samples were dissolved in ultrapure Milli-Q water at $0.8 \mathrm{mg} \mathrm{mL}^{-1}$. The results were expressed as the percentage of ACE-inhibitory activity.

\subsection{Statistical analysis}

Analysis of variance was performed using the SPSS ${ }^{\circledR}$ computer program (IBM SPSS Statistics 22 Software, Inc., Chicago, IL, USA). Differences among the samples were established using the Tukey test, with a significance level set at $p \leq 0.05$. All results are expressed as the mean of at least three replicates.

\section{Results and discussion}

\subsection{Characterization of dry powders}

3.1.1. Particle size and $\zeta$ potential. The particle sizes of both iota-carrageen-based spray-dried (SD) and heat-dried (HD) preparations containing the various natural compounds $(\mathrm{CH}, \mathrm{PP}$ and $\mathrm{SL})$ were measured after dispersing them in pure water. The results obtained for the mean size and $\zeta$ potential are shown in Table 1. As a rule, the mean particle size was noticeably lower for SD than for HD preparations. SD-CH presented a mean particle size of $3.6 \mu \mathrm{m}$, which was very close to those of spray-dried microcapsules based on maltodextrin and gum arabic containing a seed protein hydrolysate, with mean values varying between 3.3 and $6.8 \mu \mathrm{m}$ depending on the hydrolysate concentration. ${ }^{18}$ The mean particle size for SD-SL was $2.9 \mu \mathrm{m}$, which was slightly lower than the value $(5.5 \mu \mathrm{m})$ reported for spray-dried maltodextrin microcapsules with a similar shrimp lipid extract. ${ }^{19}$ The mean particle size was

Table 1 Particle properties, water solubility and entrapment efficiency (EE) of spray-dried (SD) and heat-dried (HD) preparations with collagen hydrolysate $(\mathrm{CH})$, pomegranate extract $(\mathrm{PP})$ and shrimp lipid extract $(\mathrm{SL})$

\begin{tabular}{lllll}
\hline Sample & Size $(\mu \mathrm{m})$ & $\zeta$ potential $(\mathrm{mV})$ & Water solubility $(\%)$ & EE $(\%)$ \\
\hline SD-CH & $3.60 \pm 0.04^{\mathrm{b} / \mathrm{x}}$ & $-68.05 \pm 4.20^{\mathrm{a} / \mathrm{x}}$ & $90.92 \pm 0.53^{\mathrm{a} / \mathrm{x}}$ & $96.29 \pm 0.81^{\mathrm{a} / \mathrm{x}}$ \\
SD-PP & $13.21 \pm 0.16^{\mathrm{a} / \mathrm{x}}$ & $-47.50 \pm 3.49^{\mathrm{b} / \mathrm{x}}$ & $86.49 \pm 1.51^{\mathrm{a} / \mathrm{x}}$ & $95.61 \pm 1.18^{\mathrm{a} / \mathrm{x}}$ \\
SD-SL & $2.87 \pm 0.04^{\mathrm{c} / \mathrm{x}}$ & $-47.75 \pm 2.22^{\mathrm{b} / \mathrm{x}}$ & $88.08 \pm 2.47^{\mathrm{a} / \mathrm{x}}$ & $70.36 \pm 1.54^{\mathrm{b} / \mathrm{x}}$ \\
HD-CH & $177.3 \pm 5.48^{\mathrm{c} / \mathrm{y}}$ & $-56.23 \pm 1.56^{\mathrm{a} / \mathrm{y}}$ & $36.63 \pm 2.40^{\mathrm{a} / \mathrm{y}}$ & $97.21 \pm 2.36^{\mathrm{a} / \mathrm{x}}$ \\
HD-PP & $280.3 \pm 3.32^{\mathrm{b} / \mathrm{y}}$ & $-42.90 \pm 2.24^{\mathrm{b} / \mathrm{x}}$ & $21.55 \pm 0.77^{\mathrm{b} / \mathrm{y}}$ & $97.14 \pm 1.49^{\mathrm{a} / \mathrm{x}}$ \\
HD-SL & $380.1 \pm 4.07^{\mathrm{a} / \mathrm{y}}$ & $-53.93 \pm 4.82^{\mathrm{a} / \mathrm{x}}$ & $91.23 \pm 1.02^{\mathrm{b} / \mathrm{y}}$
\end{tabular}

Different letters $(\mathrm{a}, \mathrm{b}$, and $\mathrm{c})$ indicate significance differences $(p \leq 0.05)$ among the extracts for the same dried preparation and $(\mathrm{x}$ and $\mathrm{y})$ between SD and HD preparations for the same extract. 
greater for SD-PP $(13.2 \mu \mathrm{m})$, which agrees with the range of 5.8-18.7 $\mu \mathrm{m}$ reported by Goula and Adamopoulos ${ }^{20}$ for spraydried skimmed milk powder microcapsules encapsulating a pomegranate seed extract. In contrast to SD, the HD method generally resulted in more polydispersed microparticles of several hundred micrometers (Table 1), which were clearly different in size depending on the type of compound: HD-CH were the smallest, followed in ascending order by HD-PP and HD-SL. It is important to note that not only spray drying but also heat drying resulted in small particles in the range of microns, with HD-SL being the largest microparticles with a mean size of $380 \mu \mathrm{m}$. In the HD entrapment procedure, preformed microcapsules were not dried as in typical encapsulation methods, but rather insoluble microcomplexes were formed as a result of heating, drying and subsequent grinding.

$\zeta$ potential refers to the aggregation ability of dispersed particles and is considered a key stability indicator of colloidal suspensions. The studied dispersions had the $\zeta$ potential values in the range of -42.9 and $-56.2 \mathrm{mV}$ for $\mathrm{HD}$ and -47.5 and $-68.1 \mathrm{mV}$ for SD samples (Table 1), all indicative of high particle stability. The negative charge is attributed to the anionic nature of iota-carrageenan, which typically presents a $\zeta$ potential value between -50 and $-70 \mathrm{mV}{ }^{21}$ Negative $\zeta$ potential for microcapsules obtained by Sun et $a .^{22}$ was also attributed to the employment of $\kappa$-carrageenan as the wall material.

3.1.2. Entrapment efficiency. The entrapment efficiencies (EE) of both SD and HD preparations were high in all cases, reaching values above 95\% for $\mathrm{CH}$ and $\mathrm{PP}$ samples regardless of the drying procedure used (Table 1). These values were higher than those reported, for instance, by Goula and Adamopoulos $^{20}$ for spray-dried skimmed milk powder microcapsules containing a pomegranate seed extract with an average particle size of $\approx 13 \mu \mathrm{m}(80 \%)$ or by Jayanudin et al. ${ }^{23}$ for red ginger oleoresin-chitosan-corn oil emulsions dried at $70{ }^{\circ} \mathrm{C}(79.5-82.5 \%)$. In general, comparisons with the reported literature are quite difficult since both materials and encapsulating conditions differ considerably from each other. The EE of HD-SL was also very high (91.23\%) (Table 1), but that of SD-SL was lower (70.36\%), probably because the lipids in SL were not previously emulsified or finely homogenized with the wall material solution. According to Carneiro et al., ${ }^{24}$ lipids in the form of stable emulsions typically show high encapsulation efficiency by spray-drying microencapsulation. GómezEstaca $e t ~ a l .{ }^{25}$ reported lower EE values (59.9\%) for freeze-dried gelatin-gum arabic coacervates containing a similar lipid extract from shrimp waste.

3.1.3. Water solubility. The SD powders showed high water solubility (values between 86.5 and $90.9 \%$ ), with no significant differences depending on the entrapped compound (Table 1). Besides acting as a carrier, microcapsules prepared by spray drying are known to improve the solubility of the bioactive compounds encapsulated. Similarly high water solubility was found by Zhao et al. $^{26}$ for spray-dried gelatin-dextrose microcapsules encapsulating astaxanthin (96.2\%) and by Venil et $a .^{27}$ for spray-dried $\kappa$-carrageenan microcapsules containing a flexirubin-type pigment $(91.6 \%)$. It should be noted that no extra-compound was added to the formulation to favour the crosslinking of iota-carrageenan, which could explain the high water solubility values of spray-dried microcapsules. These microcapsules could be especially useful to improve the solubility of highly hydrophobic extracts. ${ }^{28}$ In contrast, HD-CH and HD-PP presented the solubility values of $33.6 \%$ and $36.1 \%$ respectively, and HD-SL exhibited a considerably lower value $(21.6 \%)(p \leq 0.05)$, which is attributed to the lipophilic nature of SL and its lower EE. Upon drying at elevated temperature $\left(80^{\circ} \mathrm{C}\right)$ for a long time, chemical interactions between the reactive groups from both iota-carrageenan and natural compounds were favoured, leading to the formation of insoluble complexes entrapping the bioactive compounds. This kind of low-soluble microparticles could be suitable for a nutraceutical preparation, allowing the release of the entrapped bioactive after gastrointestinal digestion. In this respect, the drugpolymer insoluble complexes formed by the ionic interactions between lambda-carrageenan and diltiazem have been shown to allow controlled release of the drug under different $\mathrm{pH}$ conditions along the intestinal tract. ${ }^{10}$

3.1.4. Antioxidant activity. The antioxidant activity, determined by ABTS, FRAP and Folin reactive substances (FRS), of various natural compounds and their corresponding dried SD and HD preparations are shown in Fig. 1. For appropriate comparison between the plain compounds and dry powders, the results were expressed per gram of bioactive compound and per $g$ of dry powder. The contribution of plain carrageenan to the different antioxidant activities in both dried powders (SD and HD) was also evaluated. As reported previously regarding the ABTS values, the antioxidant activity was by far the highest in the pomegranate peel and albedo extract (PP) followed at a distance by the collagen hydrolysate. ${ }^{15}$ In particular, the high antioxidant activity of PP was attributed to its polyphenol composition, rich in $\beta$ - and $\alpha$-punicalagins, ellagic acid, rutin and epigallocatechin, while the collagen hydrolysate was mostly composed of peptides with a molecular weight lower than $1.3 \mathrm{kDa}^{15}$ The antioxidant activity of the SL extract was not measurable by any of the methods used due to its strong lipophilic nature; in this connection, considerable superoxide radical scavenging capacity as determined by the lipophilic photochemiluminiscence assay has been previously reported, which is attributed to its contents of $\alpha$-tocopherol and astaxanthin. $^{15}$

Regarding the mixture of bioactive compound and iota-carrageenan, the antioxidant activity of dried powders was generally lower than in both $\mathrm{CH}$ and PP (Fig. 1), which in part could be due to the diluting effect of the wall material. Such a diluting effect has been reported in terms of the total phenolic content for spray-dried maltodextrin microcapsules loaded with the pomegranate peel extract at different ratios. ${ }^{29}$ The only exception was $\mathrm{SD}-\mathrm{CH}$, which registered higher $(p \leq 0.05)$ FRAP values than the plain $\mathrm{CH}$ hydrolysate which indeed showed very low ferric ion reducing capacity. One possible explanation could be the capacity of iota-carrageenan to act as a reducing agent of ferric ions. ${ }^{30}$ This effect is clearly observable in Fig. 1b, where plain carrageenan SD and HD dried 

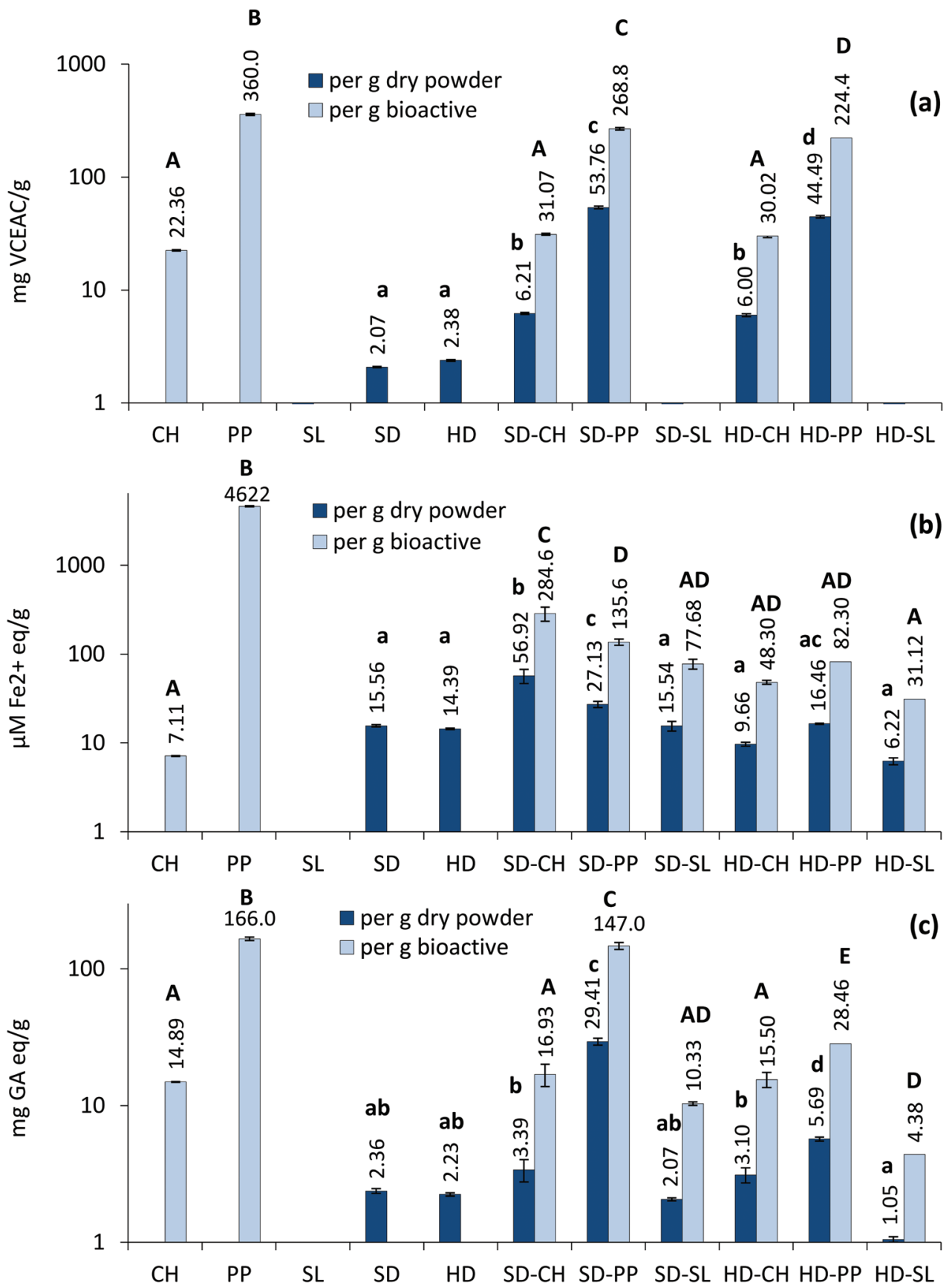

Fig. 1 Antioxidant activity of plain compounds (collagen hydrolysate $\mathrm{CH}$, pomegranate peel extract PP, and shrimp lipid extract SL), spray-dried (SD) and heat-dried (HD) iota-carrageenan, spray-dried iota-carrageenan with natural compounds (SD-CH, SD-PP and SD-SL), and heat-dried iota-carrageenan with natural compounds (HD-CH, HD-PP and HD-SL). (a) ABTS values; (b) FRAP values; (c) FRS values; expressed per g of bioactive (light bars) and per $g$ of dry powder (dark bars). Different letters $(a, b, c, \ldots)$ indicate significant differences $(p \leq 0.05)$ among the samples expressed per $g$ of dry powder. Different letters $(A, B, C, \ldots)$ indicate significant differences $(p \leq 0.05)$ among the samples expressed per $g$ of bioactive extract.

powders registered similar FRAP values than $\mathrm{CH}$. It should be noted that the FRAP values for $\mathrm{SD}-\mathrm{CH}$ were considerably higher than the sum of the values obtained for $\mathrm{CH}$ and SD separately; therefore, the possible conformational changes in peptides due to the interaction with iota-carrageenan during the atomization process should not be disregarded. Tkaczewska et $a l .{ }^{31}$ also observed an increase in the FRAP values of a carp skin gelatin hydrolysate by microencapsulation with furcellaran (a polysaccharide structurally similar to $\kappa$-carrageenan). Carrageenan also contributed to the ABTS and FRS values, but much less than for FRAP. In fact, carrageenan was probably the main factor determining the FRAP and FRS measured values for SD-SL and HD-SL dried powders, the latter showing lower values probably as a result of its lower water solubility. 
In contrast to the present work, Zhao et $a .^{26}$ reported high radical scavenging capacity in highly soluble spray-dried gelatin-maltodextrin microcapsules containing an $H$. pluvialis astaxanthin extract; this difference is probably attributed to the different wall materials used (gelatin-maltodextrin vs. iotacarrageenan) and the astaxanthin content of the original extract ( $9 \%$ vs. $0.7 \%)$.

In order to factor out the diluting effect of carrageenan in dry powders, the antioxidant activities were also expressed per $\mathrm{g}$ of bioactive agent added (Fig. 1). The antioxidant activity of the collagen hydrolysate $(\mathrm{CH})$ was maintained (by FRS and ABTS) or increased (by FRAP in SD-CH) when it was encapsulated. In contrast, HD preparations containing the pomegranate peel extract (HD-PP) registered a considerably lower $(p \leq$ 0.05 ) activity than the plain extract (Fig. 1), whether expressed as per $g$ of dry powder or per $g$ of bioactive. This effect could be due to the partial degradation of phenolic compounds during the drying process or to the possible polyphenol-polysaccharide interactions with the wall material, thus reducing the availability of the reactive groups. A similar loss of antioxidant activity (determined by ABTS) of PP was also reported in a previous work using soy phosphatidylcholine as the encapsulating agent. ${ }^{15}$ In spite of this, both SD-PP and HD-PP preparations showed the highest $(p \leq 0.05)$ activity in both ABTS and FRS assays, but the activity of SD-PP was higher ( $p \leq$ 0.05) than that of HD-PP due to its higher water solubility. Çam et $a l^{29}$ reported similar FRS values for spray-dried maltodextrin microcapsules with the pomegranate peel extract.

\subsection{Properties of surimi gels}

The addition of iota-carrageenan-based SD and HD powders containing the various natural compounds produced homogeneous and well-structured gel matrices similar to that obtained with the control gel without ingredients (Fig. 2). In contrast, the addition of plain compounds resulted in weak and friable gels, with a deformed structure, visible water syneresis and stronger coloration, especially with SL. The gels prepared with the addition of SD powders showed slightly more

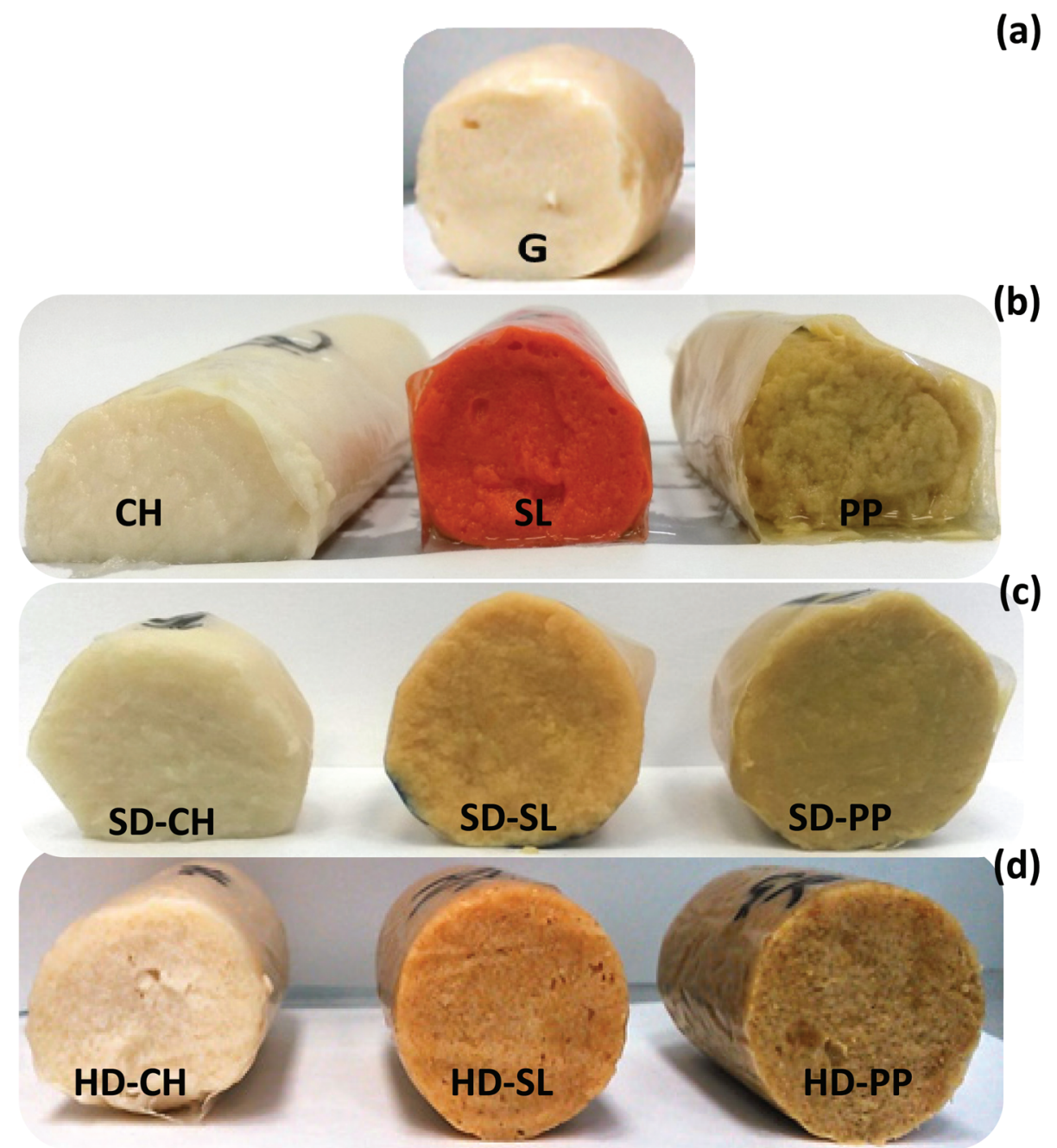

Fig. 2 Cross section of surimi gels: (a) control gel G; (b) gels with plain compounds; (c) gels with spray-dried iota-carrageenan with natural compounds (SD-CH, SD-SL and SD-PP); (d) gels with heat-dried iota-carrageenan with natural compounds (HD-CH, HD-SL and HD-PP). CH: Collagen hydrolysate; SL: shrimp lipid extract; PP: pomegranate peel extract. 
uniform cross-section than that prepared with the HD counterparts. This effect was attributed to the higher water solubility of the former, which favours homogenisation with the muscle protein. The improving effect of iota-carrageenan on the gel properties of fish mince or surimi has been well documented. ${ }^{13,14,32}$ The present work also reveals this effect by incorporating iota-carrageenan in the form of highly soluble (SD) or less soluble microparticles (HD).

3.2.1. Colour. Strong colorations of natural compounds, particularly those of SL, were considerably attenuated when they were added to the surimi gels as dry entrapment preparations (SD and HD). The Chroma and Hue colour parameters of gels incorporating SD and HD dried powders are shown in Fig. 3. No great differences were found between SD- and HDcontaining gels with the same compound, especially with the collagen hydrolysate $(\mathrm{CH})$. In fact, both $\mathrm{SD}-\mathrm{CH}$ and $\mathrm{HD}-\mathrm{CH}$ gels were very similar to the control gel (CG) due to the light coloration provided by the peptide hydrolysate. In contrast, PP and SL preparations induced more intense colour, resulting in gels with higher chroma values than the control. A slight tendency towards higher colour saturation was observed in gels formulated with SD microcapsules, likely related to their high solubility and possible compound release during the hom- ogenization step. There were slight differences in the hue values, and SD-SL and HD-SL gels stood out for showing a tendency towards greater orange coloration due to the astaxanthin content of the shrimp lipid extract. Nevertheless, the hue values in the present work denoted less orange colouration than surimi gels containing the same lipid extract encapsulated in soy phosphatidylcholine liposomes. ${ }^{15}$ In contrast, the entrapment of both $\mathrm{CH}$ and PP in iota-carrageenan did not change substantially the hue of gels as compared to their corresponding liposomal preparations.

3.2.2. Antioxidant activity. The antioxidant activities of the water soluble (non-digested) and digested (sGID) fractions of surimi gels containing both plain compounds and dry powders are shown in Fig. 4. In all tested samples, the ABTS radical scavenging capacity was noticeably higher in the digested fractions compared to the non-digested ones, including the control surimi gel (CG) without ingredients (Fig. 4a). This effect is largely attributed to the release of short antioxidant peptides resulting from the hydrolytic action of digestive enzymes (pepsin and pancreatin) on the gelled myofibrillar protein. ${ }^{33}$ The low water solubility of gels was to be expected given the formation of strong heat-stable hydrophobic and disulphide bonds during protein thermal gelation. The addition

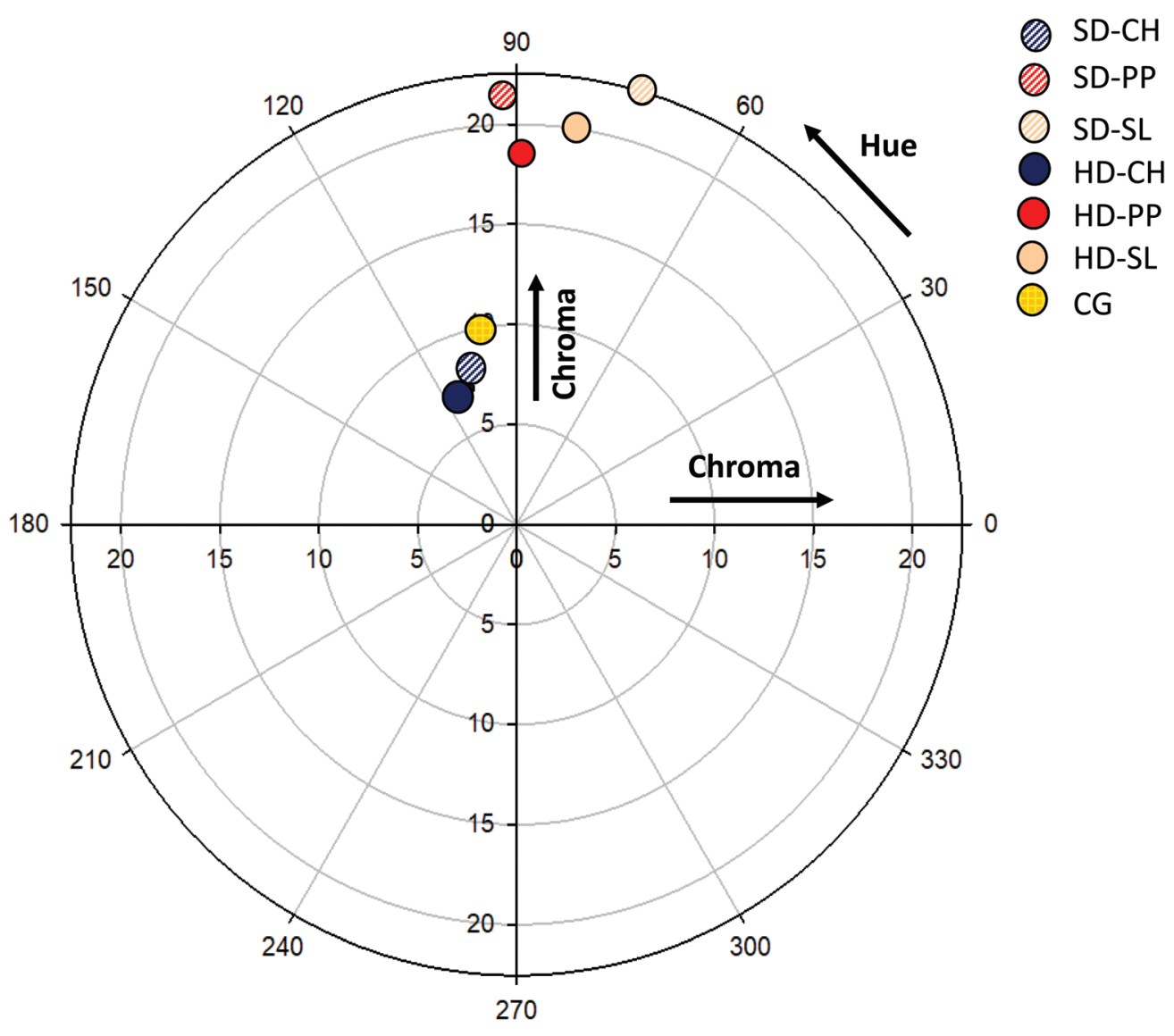

Fig. 3 Polar plot representing the saturation (Chroma) and tonality (Hue) colour parameters of gels containing spray-dried iota-carrageenan with natural compounds (SD-CH, SD-SL and SD-PP) and heat-dried iota-carrageenan with natural compounds (HD-CH, HD-SL and HD-PP). CH: Collagen hydrolysate; SL: shrimp lipid extract; PP: pomegranate peel extract. CG: Control gel. 
of natural compounds entrapped in iota-carrageenan (both SD and HD preparations) showed a general tendency to reduce the ABTS values as compared to the plain compounds in both WS and sGID fractions, which could be due to the dilution effect of the wall material with respect to the antioxidant capacity of the hydrolysed myofibrillar protein. In digested samples, the ABTS values for all gels with SD- and HD-iota-carrageenan preparations with embedded natural compounds were even lower $(p \leq 0.05)$ than in the control, suggesting that the dried powders could hinder the hydrolysis of the muscle protein. In general, surimi gels containing PP presented higher $(p \leq 0.05)$ ABTS value than those with $\mathrm{CH}$ or SL, the only exception being
HD-PP gels, which were similar to HD-CH. The contribution of PP to the radical scavenging capacity of gels, especially after SGID, was somewhat lower than would be expected in view of the noticeably high values shown by the original extract. Besides the diluting effect of PP inside the surimi gel matrix, any protein-polyphenol interactions could have reduced the activity in the extract. In addition, PP has been reported to present a considerable loss of antioxidant activity (ABTS method) when subjected to sGID, which did not occur with CH. ${ }^{15}$ As previously observed by including freeze-dried liposomes in the cited work, massive muscle protein digestion could mask the radical scavenging activity of the added com-
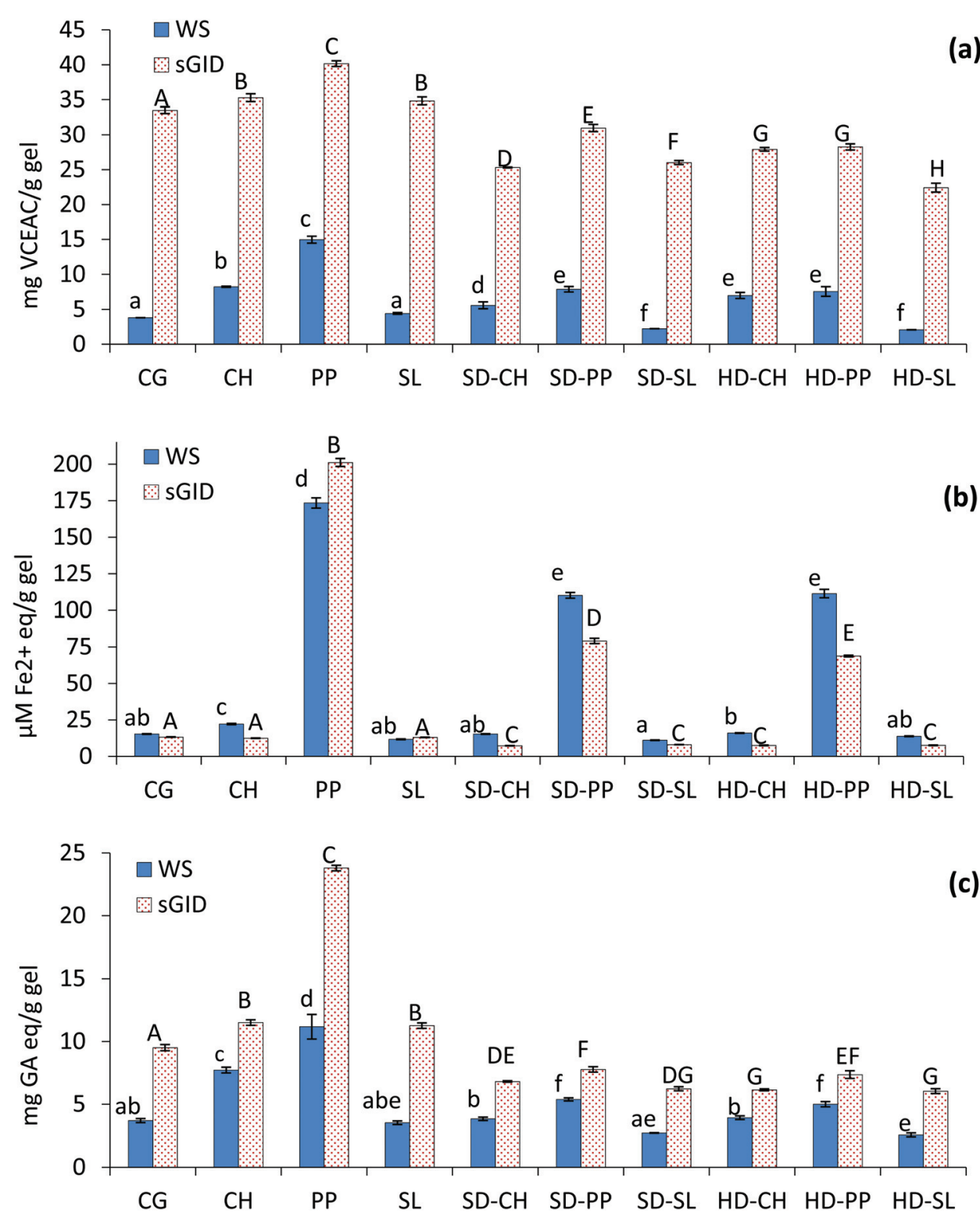

Fig. 4 Antioxidant activity of water soluble (WS) and simulated gastrointestinal digestive (sGID) fractions of surimi gels incorporating the plain compounds, spray-dried iota-carrageenan with natural compounds (SD-CH, SD-PP and SD-SL) and heat-dried iota-carrageenan with natural compounds (HD-CH, HD-PP and HD-SL). (a) ABTS values; (b) FRAP values; (c) FRS values. CH: Collagen hydrolysate; SL: shrimp lipid extract; PP: pomegranate peel extract; $C G$ : control gel. Different letters $(a, b, c, \ldots)$ indicate significant differences $(p \leq 0.05)$ among the WS fractions. Different letters $(A, B, C, \ldots)$ indicate significant differences $(p \leq 0.05)$ among the sGID fractions. 
pounds (both free or joined to iota-carrageenan), thus reducing the differences among them, regardless of their intrinsic chemical nature.

The results of ferric ion reducing capacity (FRAP) of both non-digested and digested gels are shown in Fig. 4b. Unlike ABTS, no general increase was observed in the FRAP values as a result of sGID, suggesting a negligible contribution of the digested muscle protein to the overall reducing capacity. In this case, the activity produced by PP in surimi gels was striking, especially when added as a plain extract. In contrast, $\mathrm{CH}$ and SL registered as low FRAP values as the control gel, and the values also slightly decreased when $\mathrm{CH}$ and SL were included in SD and HD preparations.

The antioxidant activity expressed as Folin reactive substances (FRS) of the different gels (Fig. 4c) showed a similar trend to that of ABTS radical scavenging activity, although in this case the rise in FRS following sGDI was less pronounced. There was a considerable increase of FRS in the gel with plain PP after SGID, so that the specific differential effect of PP was observable over and above the outcome of the digested muscle protein. This effect, however, was not evident in the corresponding dried PP preparations, where differences with respect to their HC and SL counterparts were minimal. Therefore, depending on the antioxidant mechanism studied, the activity results in gels with different compounds may vary depending on how much was contributed by the hydrolysed muscle protein.

3.2.3 Antihypertensive activity. The collagen hydrolysate $(\mathrm{CH})$ was the most antihypertensive bioactive agent with $86.9 \%$ of ACE-inhibition. It is well known that most ACE inhibitors are peptide compounds. Previous works have also reported high ACE inhibitory activity in hydrolysates from squid gelatin with Alcalase, which is attributed to the peptide sequence rich in proline residues or the enzyme used. ${ }^{16,34,35}$ In contrast, the pomegranate peel extract (PP) presented low antihypertensive activity $(24.70 \%$ of inhibition), while the lipid extract (SL) could not be quantified due to its low water solubility. When the natural compounds were subjected to SGID, the activity of $\mathrm{CH}$ slightly increased (from $86.9 \%$ to $89.9 \%$ ), while it decreased considerably in PP (from $24.7 \%$ to $7.9 \%$ ).

The ACE-inhibitory capacity of the surimi gels after sGID is shown in Fig. 5. The control gel (CG) presented 32\% of ACEinhibitory capacity, which is attributed to the release of active peptides by the hydrolysis of surimi proteins. The addition of any of the plain compounds led to a significant increase in the ACE-inhibition percentage, the gel with plain $\mathrm{CH}$ being the sample with highest activity (71.2\%). It is worth noting the augmented ( $p \leq 0.05)$ activity in the gels containing plain PP $(63.87 \%)$ and SL $(50.9 \%)$ compared to the control gel. These extracts did not stand out for their high ACE-inhibition, and thus the measured activity, especially with SL, should be attributed to their interference in protein gelation, causing the muscle protein to be more exposed to digestive enzymes. As mentioned before, good gels were not obtained by the addition of plain compounds. The ACE-inhibitory activity of gels containing SD-CH and HD-CH was lower than that of gels with the plain hydrolysate, but higher than that of the control gel, despite the diluting effect and gelling capacity of iota-carrageenan. A similar finding, but with lower values, was also observed with SD-PP. The presence of iota-carrageenan in the gel matrix would also affect the protein-protein interactions, and therefore muscle protein digestion. However, the resulting ACE-inhibitory activity varied considerably depending on the dried preparation, since HD-PP, SD-SL and HD-SL led to gels with ACE-inhibition values lower than the control gel. So, the observed differences could be attributed to the activity provided by the natural compounds, which was considerably higher with $\mathrm{CH}$, especially when entrapped in the more soluble form (SD-CH). In the case of SD-PP, the encapsulation process could have protected the activity of PP from digestion, while the low solubility and/or lack of activity in HD-PP, SD-SL and HD-SL preparations might have contributed to the decrease of the ACE-inhibitory potential of the hydrolysed myofibrillar protein.

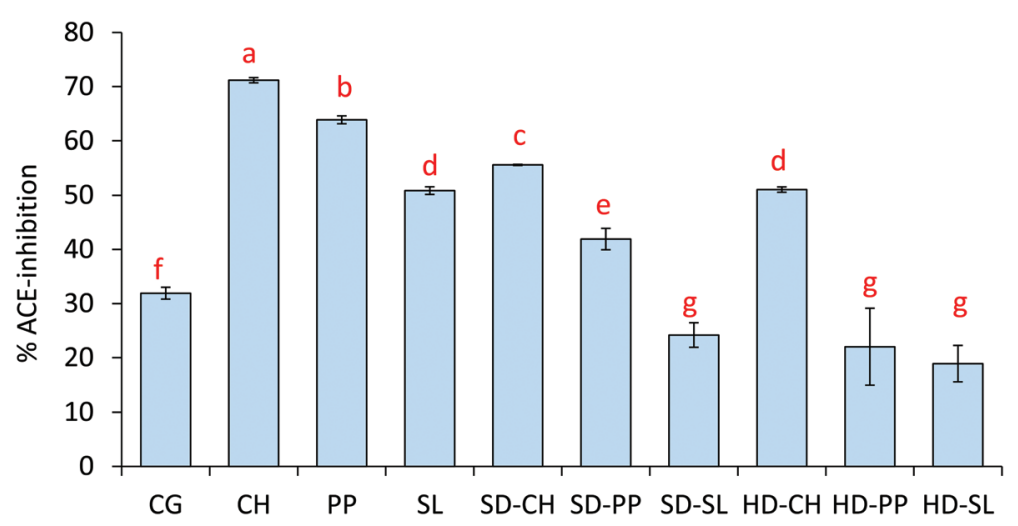

Fig. 5 Antihypertensive activity, expressed as percentage of ACE inhibition, after simulated gastrointestinal digestion of surimi gels incorporating the plain compounds, spray-dried iota-carrageenan with natural compounds (SD-CH, SD-PP and SD-SL) and heat-dried iota-carrageenan with natural compounds (HD-CH, HD-PP and HD-SL). CH: Collagen hydrolysate; SL: shrimp lipid extract; PP: pomegranate peel extract; CG: control gel. Different letters $(a, b, c, \ldots)$ indicate significant differences $(p \leq 0.05)$ among the samples. 


\section{Conclusion}

Both spray-drying (SD) and heat-drying (HD) of the aqueous dispersions of iota-carrageenan and natural food waste compounds resulted in antioxidant microparticle dry powders, which showed good miscibility with surimi protein to obtain well-conformed thermal gels, regardless of different solubilities of both types of preparations. Both types of dry powders were then suitable for addition as technological ingredients to surimi gel formulations. The noticeable high antioxidant capacity of the pomegranate extract (PP) was considerably decreased when included via SD or HD preparations, while the entrapped SL extract presented a limited antioxidant power, despite becoming highly soluble in the SD preparation. In general, the antioxidant properties and ACE-inhibitory capacity of the surimi gels determined after in vitro gastrointestinal digestion were noticeably lower when the natural compounds were entrapped in the iota-carrageenan matrices regardless of the drying method used, suggesting that the technological functionality of the dry powders could be superior to the bioactive itself. In vivo studies would be necessary to overcome possible limitations derived from the presence of iota-carrageenan, as well as its possible functionality as a dietary fibre.

\section{CRediT author statement}

Daniel Marín-Peñalver: Formal analysis; investigation; methodology; writing original draft; Ailén Alemán: Formal analysis; investigation; methodology; M. Pilar Montero: Conceptualization; methodology; resources; supervision; project administration; funding acquisition; and $\mathrm{M}$. Carmen Gómez-Guillén: Conceptualization; methodology; resources; supervision; project administration; funding acquisition; writing review and editing.

\section{Conflicts of interest}

There are no conflicts of interest to declare.

\section{Acknowledgements}

The authors are grateful for the financial support provided by Agencia Estatal de Investigación (AEI) and Fondo Europeo de Desarrollo Regional (FEDER) through the project NANOALIVAL AGL2017-84161 and by CSIC through the project 202070E218. We acknowledge support of the publication fee by the CSIC Open Access Publication Support Initiative through its Unit of Information Resources for Research (URICI).

\section{References}

1 M. G. Bah, H. M. Bilal and J. Wang, Fabrication and application of complex microcapsules: A review, Soft Matter, 2020, 16(3), 570-590.
2 M. Saifullah, M. R. I. Shishir, R. Ferdowsi, M. R. T. Rahman and Q. V. Vuong, Micro and nano encapsulation, retention and controlled release of flavor and aroma compounds: A critical review, Trends Food Sci. Technol., 2019, 86, 230-251.

3 J.-H. Ye and M. A. Augustin, Nano- and micro-particles for delivery of catechins: Physical and biological performance, Crit. Rev. Food Sci. Nutr., 2019, 59(10), 1563-1579.

4 F. Nazzaro, P. Orlando, F. Fratianni and F. Coppola, Microencapsulation in food science and technology, Curr. Opin. Biotechnol., 2012, 23, 182-186.

5 C. Encina, C. Vergara, B. Giménez, F. Oyarzún-Ampuero and P. Robert, Conventional spray-drying and future trends for the microencapsulation of fish oil, Trends Food Sci. Technol., 2016, 56, 46-60.

6 E. Assadpour and S. M. Jarari, Advances in Spray-Drying Encapsulation of Food Bioactive Ingredients: From Microcapsules to Nanocapsules, Annu. Rev. Food Sci. Technol., 2019, 10, 103-131.

7 L. Gasperini, J. F. Mano and R. L. Reis, Natural polymers for the microencapsulation of cells, J. R. Soc., Interface, 2014, 11(100), 0817.

8 T. R. Thrimawithana, S. Young, D. E. Dunstan and R. G. Alany, Texture and rheological characterization of kappa and iota carrageenan in the presence of counter ions, Carbohydr. Polym., 2010, 82(1), 69-77.

9 H. He, J. Ye, X. Zhang, Y. Huang, X. Li and M. Xiao, $\kappa$-Carrageenan/locust bean gum as hard capsule gelling agents, Carbohydr. Polym., 2017, 175, 417-424.

10 M. C. Bonferoni, S. Rossi, F. Ferrari, G. P. Bettinetti and C. Caramella, Characterization of a diltiazem-lambda carrageenan complex, Int. J. Pharm., 2000, 200, 207-216.

11 L. Mao, Q. Pan, Z. Hou, F. Yuan and Y. Gao, Development of soy protein isolate-carrageenan conjugates through Maillard reaction for the microencapsulation of Bifidobacterium longum, Food Hydrocolloids, 2018, 84, 489497.

12 S. Ścieszka and E. Klewicka, Algae in food: a general review, Crit. Rev. Food Sci. Nutr., 2019, 59(21), 3538-3547.

13 M. Pérez-Mateos, T. Solas and P. Montero, Carrageenans and alginate effects on properties of combined pressure and temperature in fish mince gels, Food Hydrocolloids, 2002, 16(3), 225-233.

14 C. Gómez-Guillen, T. Solas, J. Borderías and P. Montero, Effect of heating temperature and sodium chloride concentration on ultrastructure and texture of gels made from giant squid (Dosidicusgigas) with addition of starch, 1-carrageenan and egg white, Z. Lebensm.-Unters. Forsch., 1996, 202(3), 221-227.

15 D. Marín, A. Alemán, A. Sánchez-Faure, P. Montero and M. C. Gómez-Guillén, Freeze-dried phophatidylcholine liposomes encapsulating various antioxidant extracts from natural waste as functional ingredients in surimi gels, Food Chem., 2018, 245, 525-535.

16 A. Alemán, B. Giménez, E. Pérez-Santín, M. C. GómezGuillén and P. Montero, Contribution of Leu and Hyp residues to antioxidant and ACE-inhibitory activities of peptide 
sequences isolated from squid gelatin hydrolysate, Food Chem., 2011, 125, 334-341.

17 K. Slinkard and V. L. Singleton, Total phenol analysis: automation and comparison with manual methods, Am. J. Enol. Vitic., 1977, 28(1), 49-55.

18 R. E. Cian, A. Campos-Soldini, L. Chel-Guerrero, S. R. Drago and D. Betancur-Ancona, Bioactive Phaseoluslunatus peptides release from maltodextrin/gum arabic microcapsules obtained by spray drying after simulated gastrointestinal digestion, Int. J. Food Sci. Technol., 2019, 54, 2002-2009.

19 P. Montero, M. M. Calvo, M. C. Gómez-Guillén and J. Gómez-Estaca, Microcapsules containing astaxanthin from shrimp waste as potential food coloring and functional ingredient: Characterization, stability and bioaccessibility, LWT-Food Sci. Technol., 2016, 70, 229-236.

20 A. M. Goula and K. G. Adamopoulos, A method for pomegranate seed application in food industries: Seed oil encapsulation, Food Bioprod. Process., 2012, 90, 639-652.

21 S. David, A. Wojciechowska, R. Portmann, A. Shpigelman and U. Lesmes, The impact of food-grade carrageenans and consumer age on the in vitro proteolysis of whey proteins, Food Res. Int., 2020, 108964.

22 X. Sun, C. Pan, Z. Ying, D. Yu, X. Duan, F. Huang, J. Ling and X. K. Ouyang, Stabilization of zein nanoparticles with $\kappa$-carrageenan and tween 80 for encapsulation of curcumin, Int. J. Biol. Macromol., 2020, 146, 549-559.

23 Jayanudin, M. Fahrurrozi and S. K. Wirawan, Rochmadi. Antioxidant activity and controlled release analysis of red ginger oleoresin (Zingiber officinale var. Rubrum) encapsulated in chitosan cross-linked by glutaraldehyde saturated toluene, Sustainable Chem. Pharm., 2019, 12, 100132.

24 H. C. F. Carneiro, R. V. Tonon, C. R. F. Grosso and M. D. Hubinger, Encapsulation efficiency and oxidative stability of flaxseed oil microencapsulated by spray drying using different combinations of wall materials, J. Food Eng., 2013, 115(4), 443-451.

25 J. Gómez-Estaca, T. A. Comunian, P. Montero, R. FerroFurtado and C. S. Favaro-Trindade, Encapsulation of an astaxanthin-containing lipid extract from shrimp waste by complex coacervation using a novel gelatin-cashew gum complex, Food Hydrocolloids, 2016, 61, 155-162.
26 X. Zhao, H. Liu, X. Zhang, G. Zhang and H. Zhu, Astaxanthin from Haematococcuspluvialis Microencapsulated by Spray Drying: Characterization and Antioxidant Activity, J. Am. Oil Chem. Soc., 2019, 96, 93-102.

27 C. K. Venil, A. R. Khasim, C. A. Aruldass and W. A. Ahmad, Microencapsulation of flexirubin-type pigment by spray drying: Characterization and antioxidant activity, Int. Biodeterior. Biodegrad., 2016, 113, 350-356.

28 S. W. Chan, H. Mirhosseini, F. S. Taip, T. C. Ling, I. A. Nehdi and C. P. Tan, Emulsion Formulation Optimization and Characterization of Spray-dried $\kappa$-Carrageenan Microparticles for the Encapsulation of CoQ10, Food Technol. Biotechnol., 2016, 25(S), 53-62.

29 M. Çam, N. C. İçyer and F. Erdoğan, Pomegranate peel phenolics: Microencapsulation, storage stability and potential ingredient for functional food development, LWT-Food Sci. Technol., 2014, 55, 117-123.

30 E. Sokolova, A. Barabanova, R. Bogdanovich, V. Khomenko, T. Soloveva and I. Yermak, In vitro antioxidant properties of red algal polysaccharides, Biomed. Pharmacother., 2011, 1, 161-167.

31 J. Tkaczewska, E. Jamróz, E. Piątkowska, B. Borczak, J. Kapusta-Duch and M. Morawska, Furcellaran-Coated Microcapsules as Carriers of Cyprinuscarpio Skin-Derived Antioxidant Hydrolysate: An In Vitro, and In Vivo Study, Nutrients, 2019, 11, 2502.

32 A. Hunt and J. W. Park, Alaska Pollock Fish Protein Gels as Affected by Refined Carrageenan and Various Salts, J. Food Qual., 2013, 36(1), 51-58.

33 N. Ueki, J. Wan and S. Watabe, The Pepsin Digestibility of Thermal Gel Products Made from White Croaker (Pennahia argentata) Muscle in Associating with Myosin Polymerization Levels, J. Food Sci., 2014, 79(12), C2427C2433.

34 A. Alemán, E. Pérez-Santín, S. Bordenave-Juchereau, I. Arnaudin, M. C. Gómez-Guillén and P. Montero, Squid gelatin hydrolysate with antihypertensive, anticancer and antioxidant activity, Food Res. Int., 2011b, 44(4), 1044-1051.

35 A. Alemán, M. C. Gómez-Guillén and P. Montero, Identification of ace-inhibitory peptides from squid skin collagen after in vitro gastrointestinal digestion, Food Res. Int., 2013, 54(1), 790-795. 